\title{
Clinical Profile and Outcome and Risk Factors of Cardiovascular Involvement in Hospitalized Children with Kawasaki Disease
}

\author{
A. K. Al-Miraj ${ }^{1 *}$, S. M. Rezwanuzzaman ${ }^{2}$, Sakera Khatun Mony ${ }^{3}$, Muhammad Abduz Zaher ${ }^{4}$, Md. Rezaul Alam ${ }^{5}$, \\ Mohammad Ata Ullah ${ }^{6}$, Sharmin Akter ${ }^{7}$, Mohammad Maruf Reza $^{8}$ \\ ${ }^{1}$ Research Assistant, Department of Vascular surgery, Bangabandhu Sheikh Mujib Medical University, Dhaka, Bangladesh \\ ${ }^{2}$ Medical Officer, Internal Medicine Department, Bangabandhu Sheikh Mujib Medical University, Dhaka, Bangladesh \\ ${ }^{3}$ Research Fellow at Institute of nutrition and food science University Of Dhaka Bangladesh \\ ${ }^{4}$ Associate Professor, Institute of Nutrition and Food Science University of Dhaka Bangladesh \\ ${ }^{5}$ Assistant Professor, Department of Cardiology, M. Abdur Rahim Medical College, Dinajpur, Bangladesh \\ ${ }^{6}$ Assistant Professor, Department of Cardiac Surgery, Bangabandhu Sheikh Mujib Medical University, Dhaka, Bangladesh \\ ${ }^{7}$ Medical Assistant, E-Z Medical Care, 85-50 118th Street, Kew Gardens, NY-11415, United States \\ ${ }^{8}$ Quality Analyst (Endocrinology), Central Queens IPA, United States \\ *Corresponding Author: A. K. Al-Miraj \\ Research Assistant, Department of Vascular surgery, Bangabandhu Sheikh Mujib Medical University, Dhaka, Bangladesh
}

Article History: | Received: 16.12.2021 | Accepted: 23.01.2022 | Published: 28.02.2022 |

\begin{abstract}
Kawasaki disease (KD), also known as Kawasaki syndrome, is an acute febrile illness of unknown cause that primarily affects children younger than 5 years of age. The present study was conducted to study the clinical profile and outcome and risk factors of Kawasaki disease in children. This observational prospective study was conducted in cardiology ward with a diagnosis of Kawasaki disease from $1^{\text {st }}$ June 2020 to 31 July 2021 in Bangabandhu Sheikh Mujib Medical University Hospital Dhaka, Bangladesh. Forty five (45) children aged between 3 months to 10 years were studied. Kawasaki disease is more prevalent among male child (73.33\%). Most commonly encountered risk factor for cardiovascular involvement especially coronary dilatation or aneurysmal change is more observed in younger age of presentation. Fever, rash, conjunctivitis, erythema and edema of limbs, periungual skin desquamations are the presenting features. Cardiovascular involvement of left main coronary artery dilation is the most frequently observed aneurismal change observed among study population which was evident in $68 \%$ of the children having coronary involvement. All the study patient received high dose aspirin (100\%) and 93\% received IVIG of whom $6.66 \%$ had IVIG- resistant Kawasaki disease. Commonly occurring complication is uveitis (17.77\%). Among the total 45 study population 1 patient expired (2.22\%).Clinicians should have a high index of suspicion of Kawasaki disease in persistently febrile patients and once clinically diagnosed, echocardiography should be done and IVIG therapy along with aspirin should be started, specially in infants $(<6$ months $)$ as they have the higher tendency to develop coronary aneurysm.

Keywords: Characteristics, Cardiovascular Involvement, Aneurysm, IVIG, Treatment, Outcome.

Copyright () 2022 The Author(s): This is an open-access article distributed under the terms of the Creative Commons Attribution 4.0 International License (CC BY-NC 4.0) which permits unrestricted use, distribution, and reproduction in any medium for non-commercial use provided the original author and source are credited.
\end{abstract}

\section{INTRODUCTION}

Kawasaki disease (KD), also known as Kawasaki syndrome, is an acute febrile illness of unknown cause that primarily affects children younger than 5 years of age [1]. The disease was first described in Japan by Tomisaku Kawasaki in 1967, and the first cases outside of Japan were reported in Hawaii in 1976 [2]. Clinical signs include fever, rash, swelling of the hands and feet, irritation and redness of the whites of the eyes, swollen lymph glands in the neck, and irritation and inflammation of the mouth, lips, and throat. The disease usually occurs in infants and children under 5 years [3, 4]. Although one or more infectious triggers are most likely, the precise etiology is still unknown. There is no specific diagnostic test or pathognomonic clinical feature and still diagnosis of $\mathrm{KD}$ is made on clinical criteria [5]. Children with Kawasaki disease might have high fever, swollen hands 
and feet with skin peeling, and red eyes and tongue. But Kawasaki disease is usually treatable, and most children recover without serious problems if they receive treatment within 10 days of onset. The diagnostic criteria includes simultaneous presence of high grade fever for 5 or more days with at least 4 out of 5 symptoms (bilateral non-exudative bulbar conjunctivitis, polymorphous exanthema, erythema of lips and oral cavity, edema and desquamation of extremities and cervical lymphadenopathy) or fever associated with less than 4 of the diagnostic criteria and echocardiographic abnormalities of the coronary arteries [6]. Other manifestations like arthritis or arthralgia, diarrhea, vomiting, abdominal pain, irritability, aseptic meningitis, aseptic pyuriaetc may be present [7]. In children younger than 6 months, the diagnosis of Kawasaki disease is quite difficult as the presentation is usually atypical or not fulfilling the clinical criteria and it accounts $15-20 \%$ of all patient [8]. Therefore, a high index of suspicion is needed for any infant or child with fever of unknown origin, in order to avoid a missed or delayed diagnosis.

\section{MATERIALS AND METHODS}

This study is an observational prospective study. This observational prospective study was conducted in cardiology ward with a diagnosis of Kawasaki disease from $1^{\text {st }}$ June 2020 to 31 July 2021 in Bangabandhu Sheikh Mujib Medical University Hospital Dhaka, Bangladesh. The diagnosis of KD was made when a child of any age- group presented with unexplained fever $\left(>38^{\circ} \mathrm{C}\right)$ for at least five days and four of the following: (i) bilateral conjunctival congestion without exudate, (ii) changes of the oral mucous membrane (any 1): congested pharynx, congested/fissured lips, strawberry tongue, (iii) Polymorphous rash, (iv) changes of the extremities: desquamation or edema, and (v) unilateral lymphadenopathy [9]. Demographic data and base-line characteristics were collected and a structured history was taken. Baseline laboratory work-up included hemoglobin, white cell counts, and inflammatory markers like CRP and/or ESR and transthoracic echocardiography (TTE) using Vivid E9. Parasternal long- and short-axis windows, as well as apical fourand two-chamber views, were used to obtain twodimensional evaluations, M-mode dimensions and duplex Doppler studies. Additional tests like urine microscopy, urine/blood culture, renal and liver function test were done based on clinical circumstances. Specific attention was paid to potential risk factors. During the physical examination, the presence or absence of any vascular phenomena was also sought. Upon diagnosis, patients received intravenous immunoglobulin (IVIG) (2 g/kg) infused over 10-12 hours and oral aspirin $(80 \mathrm{mg} 100 \mathrm{mg} / \mathrm{kg} / \mathrm{d})$. IVIG- resistant KD was defined as persistent or recrudescent fever 36 hours after the completion of the initial immunoglobulin infusion. Data were analyzed using SPSS software version 19.

\section{RESULTS}

Table I: Sex and Age distribution of the study patients $(n=45)$

\begin{tabular}{|c|c|c|}
\hline \\
\hline & n & $\%$ \\
\hline \multicolumn{3}{|l|}{ Sex } \\
\hline Male & 33 & 73.3 \\
\hline Female & 12 & 26.7 \\
\hline \multicolumn{3}{|l|}{ Age } \\
\hline$>6$ months & $4(\mathrm{M}-3 ; \mathrm{F}-1)$ & $8.88 \%$ \\
\hline 6months-<1yr & $3(\mathrm{M}-3 ; \mathrm{F}-0)$ & $6.6 \%$ \\
\hline $1 \mathrm{yr}-<2 \mathrm{yr}$ & $10(\mathrm{M}-8 ; \mathrm{F}-2)$ & $22.22 \%$ \\
\hline $2 \mathrm{yr}-<3 \mathrm{yr}$ & $8(\mathrm{M}-6 ; \mathrm{F}-2)$ & $17.77 \%$ \\
\hline $3 \mathrm{yr}-<4 \mathrm{yr}$ & $4(\mathrm{M}-4 ; \mathrm{F}-0)$ & $8.88 \%$ \\
\hline $4 \mathrm{yr}-<5 \mathrm{yr}$ & $4(\mathrm{M}-2 ; \mathrm{F}-2)$ & $8.88 \%$ \\
\hline $5 \mathrm{yr}-<7 \mathrm{yr}$ & $5(\mathrm{M}-3 ; \mathrm{F}-2)$ & $11.11 \%$ \\
\hline $7 \mathrm{yr}-<9 \mathrm{yr}$ & $4(\mathrm{M}-2 ; \mathrm{F}-2)$ & $8.88 \%$ \\
\hline$>9 \mathrm{yr}$ & $3(\mathrm{M}-2 ; \mathrm{F}-1)$ & $6.6 \%$ \\
\hline
\end{tabular}

In this study out of total 45 study population $73.3 \%(\mathrm{n}=33)$ were male and $26.7 \%(\mathrm{n}=12)$ were female. Mean patient age was $3 \mathrm{yr}-<4 \mathrm{yr}$ (range, 3 months to 10 years), and $4(8.88 \%)$ patients were $<6$ months of age, $3(6.6 \%)$ patients were between 6 month to $<1$ year and $>9$ years respectively, $10(22.22 \%)$ patients were 1years to $<2$ yrs., $8(17.77 \%)$ are between 2 to $3 y$ rs, $4(8.88 \%)$ were respectively between 3 to 4 years and 4 to 5 years and between 7 to 9 years, $5(11.11 \%)$ between 5 to 7 yrs. of age (Table-1).

Table-2: Distribution of provisional/referral diagnosis $(n=45)$

\begin{tabular}{|l|l|l|}
\hline Initial diagnosis & $\mathbf{n}$ & $\mathbf{\%}$ \\
\hline Suspected Leukemia & 2 & 4.44 \\
\hline Pyrexia of unknown origin & 19 & 42.22 \\
\hline Heart failure & 3 & 6.66 \\
\hline Enteric fever & 6 & 13.33 \\
\hline Septicemia & 10 & 22.22 \\
\hline Hepatitis & 3 & 6.66 \\
\hline Meningitis & 1 & 2.22 \\
\hline
\end{tabular}

None of the under six month patients were neonates. The trend is gradual increment in the number of diagnosed patients of Kawasaki disease every year. This study shows provisional or referral diagnosis of $42.22 \%(n=19)$ were Pyrexia of unknown origin and enteric fever $13.33 \%(n=6)$. The other provisional diagnoses are septicemia $22.22 \%(n=10)$, heart failure and hepatitis both $6.66 \%(\mathrm{n}=3)$, suspected leukemia $4.44 \%(n=2)$ and meningitis $2.22 \%(n=1)($ Table-2). 
Table-3: Presenting features of the patient (multiple response) $(n=45)$

\begin{tabular}{|l|l|l|}
\hline Presenting feature & n & \% \\
\hline Fever(>5 days) & 45 & 100 \\
\hline Skin rash (polymorphous) & 26 & 57.77 \\
\hline Non purulent conjunctivitis & 44 & 97.7 \\
\hline $\begin{array}{l}\text { Changes in the lips and oral cavity(lip cracking, } \\
\text { strawberry tongue, erythema) }\end{array}$ & 43 & 95.5 \\
\hline Erythema of palms and soles & 37 & 82.22 \\
\hline Edema of hands and feet & 36 & 80.0 \\
\hline Periungual peeling & 32 & 71.11 \\
\hline Perianal desquamation & 35 & 77.77 \\
\hline Cervical lymphadenopathy(unilateral, >1.5cm) & 23 & 51.11 \\
\hline Hepatomegaly & 5 & 11.11 \\
\hline Arthritis/arthralgia & 6 & 13.33 \\
\hline Asceptic meningitis & 1 & 2.22 \\
\hline Uveitis & 7 & 15.55 \\
\hline Pyuria(sterile) & 4 & 8.88 \\
\hline Mean duration of fever 12 \pm 3 days & \multicolumn{2}{|l}{} \\
\hline
\end{tabular}

This study also shows all the 45 patients $(100 \%)$ had fever. They also presented with conjunctivitis $(97.7 \%)$, changes in oral mucosa $(95.5 \%)$, erythema of palm and soles $(82.22 \%)$, edema of hand and feet $(80.0 \%)$, perianal desquamation $(77.77 \%)$ and periungual peeling of skin $(71.11 \%)$. The other presentations were cervical lymphadenopathy $(51.11 \%)$, skin rash $(57.77 \%)$, arthritis, uveitis, hepatomegaly, sterile pyuria and aseptic meningitis (Table-3).

Table-4: Distribution of Laboratory data (multiple response) $(n=45)$

\begin{tabular}{|l|l|l|}
\hline Laboratory data & Frequency (number) & Percentage (\%) \\
\hline Anaemia & 30 & $66.66 \%$ \\
\hline Leukocytosis(WBC count $\left.>15,000 / \mathrm{mm}^{3}\right)$ & 35 & $77.77 \%$ \\
\hline Neutrophils $(\%)(>70 \%)$ & 40 & $88.8 \%$ \\
\hline Thrombocytosis(Platelet count $\left.>450000 / \mathrm{mm}^{3}\right)$ & 31 & $72 \%$ \\
\hline Raised ESR $(>50 \mathrm{~mm} / \mathrm{h})$ & 39 & $86.66 \%$ \\
\hline Raised CRP(>6 mg/dL) & 33 & $76.7 \%$ \\
\hline Blood culture positive & 1 & $2.2 \%$ \\
\hline S. ALT(>45U/L) & 20 & $44.44 \%$ \\
\hline Raised S.creatinine $(>100 \mu \mathrm{mol} / \mathrm{L})$ & 1 & $2.2 \%$ \\
\hline Abnormal Lipid profile & 2 & $4.44 \%$ \\
\hline
\end{tabular}

In this study majority of patients had neutrophilic leukocytosis ( $\mathrm{n}=40$ 0r $88.88 \%$ ), raised ESR $(\mathrm{n}=39$ or $86.66 \%)$. Raised CRP and leukocytosis were found in $77.77 \% \quad(n=35)$. Anemia was observed in
$66.66 \%$ patient. Positive blood culture and raised serum creatinine was found in $2.2 \%$ of patients respectively; $4.44 \%$ patient $(n=2)$ had abnormal lipid profile (Table4).

Table-5: Distribution of echocardiographic data (multiple response) $(n=45)$

\begin{tabular}{|l|l|l|l|}
\hline $\begin{array}{l}\text { Echocardiography } \\
\text { findings }\end{array}$ & Artery involved & $\begin{array}{l}\text { No of cases (\%) } \\
(\mathbf{n = 1 9})\end{array}$ & Mean Size (mm) \\
\hline \multirow{4}{*}{ Change in artery or aneurysms } & Left main coronary artery (LMCA) & $68 \%(\mathrm{~N}=13)$ & $2.9 \pm 0.47$ \\
\cline { 2 - 4 } & Left anterior descending artery (LAD) & $52 \%(\mathrm{n}=10)$ & $2.8 \pm 0.43$ \\
\cline { 2 - 4 } & Left circumflex artery (LCX) & $10.5 \%(\mathrm{n}=2)$ & $2.2 \pm 0.11$ \\
\cline { 2 - 4 } & Right coronary artery (RCA) & $36.8 \%(\mathrm{n}=7)$ & $3.2 \pm 0.41$ \\
\cline { 2 - 4 } & Both LAD and LCX & $15.7 \%(\mathrm{n}=3)$ & $3.0 \pm 0.32$ \\
\cline { 2 - 4 } & LMCA, LAD, LCX and RCA & $10.5 \%(\mathrm{n}=2)$ & $3.1 \pm 0.21$ \\
\hline
\end{tabular}

Table-6: Distribution of other echocardiographic findings (multiple response) $(n=45)$

\begin{tabular}{|l|l|}
\hline Other echocardiography findings & No of cases(n=19) \\
\hline Pericardial effusion & 6 \\
\hline LV dysfunction & 3 \\
\hline Mitral regurgitation & 2 \\
\hline Tricuspid regurgitation & 2 \\
\hline
\end{tabular}


Echocardiographic evaluation shows among the 19 patients having coronary change majority that is $68 \%$ had isolated left main coronary artery (LMCA) dilatation with mean size $2.9 \pm 0.47(\mathrm{~mm})$. Second highest range of dilatation was observed in right coronary artery (RCA) with mean size of dilatation $3.2 \pm 0.41(\mathrm{~mm})$. Both LAD and LCX dilatation was found in $15.7 \%$ children with mean size of $3.0 \pm 0.32$ $(\mathrm{mm})$. All 4 coronary vessels (LMCA, LAD, LCX and RCA) were found dilated in 2 patient $(10.5 \%)$. Other than coronary dilatation, 6 patient out of 19 patients had pericardial effusion, 3 had left ventricular dysfunction and 2 patient each had mitral regurgitation and tricuspid regurgitation (Table-5, 6).

Table-7: Treatment options $(n=45)$

\begin{tabular}{|l|l|l|}
\hline Treatment & $\mathrm{n}$ & $\%$ \\
\hline Intravenous immunoglobulin(IVIG) & 42 & $93 \%$ \\
\hline Requiring second dose of IVIG & 3 & $6.9 \%$ \\
\hline High dose Aspirin(80-100mg/kg) in acute phase & 45 & $100 \%$ \\
\hline Antiplatelet drug(Clopedogril) & 2 & $4.44 \%$ \\
\hline Anti-coagulant( Warfarin) & 5 & $11.11 \%$ \\
\hline 20\% human albumin & 1 & $2.22 \%$ \\
\hline
\end{tabular}

This study shows treatment received by the patients of this study. Out of 45 of the study patient, 42 patient $(93 \%)$ received at least single dose of IVIG @ $2 \mathrm{gm} / \mathrm{kg}$ over the period of $10-12$ hours. $65 \%$ of patient received IVIG within 12 days of onset of fever and rest of the patient, that is $35 \%$ patient received IVIG after this period. Among the three patients who did not received IVIG, two patient left hospital against medical advice (LAMA) and one patient expired. Again out of the 40 patient who received initial dose of IVIG required $2^{\text {nd }}$ dose of IVIG due to recurrence of fever or IVIG resistant KD after 36 hours after initial IVIG therapy (Table-7). All the 45 study patient received high dose of aspirin ( $80-100 \mathrm{mg} / \mathrm{kg} /$ day) in the initial stage and was switched to lower dose $(3-5 \mathrm{mg} / \mathrm{kg} / \mathrm{day})$ for only antiplatelet activity for 6 weeks or longer. However 5(11.11\%) patient required anticoagulant drug that is warfarin. Two patient were given additional antiplatelet agent as clopedogril and one patient required 20\% human albumin infusion (Table-7).

Table-8: Complications and outcome $(n=45)$

\begin{tabular}{|l|l|l|l|}
\hline \multirow{4}{*}{ Complications } & & $\mathrm{n}$ & $\%$ \\
\cline { 2 - 4 } & IVIG resistant Kawasaki & 3 & $6.66 \%$ \\
\cline { 2 - 4 } & Acute kidney injury & 1 & $2.22 \%$ \\
\cline { 2 - 4 } & Hepatitis & 4 & $8.88 \%$ \\
\cline { 2 - 4 } & Uveitis & 7 & $15.55 \%$ \\
\cline { 2 - 4 } & Asceptic meningitis & 1 & $2.22 \%$ \\
\cline { 2 - 4 } & Convulsion & 2 & $4.44 \%$ \\
\cline { 2 - 4 } & Hearing loss & 1 & $2.22 \%$ \\
\hline Outcome & Improved & 39 & $86 \%$ \\
\cline { 2 - 4 } & Requiring second dose of IVIg & 3 & $6.66 \%$ \\
\cline { 2 - 4 } & Discharged against medical advice & 2 & $4.44 \%$ \\
\cline { 2 - 4 } & Died & 1 & $2.22 \%$ \\
\hline
\end{tabular}

The complications observed in the study populations were recurrent Kawasaki in 3(6.66\%) patient, acute kidney injury in $1(2.22 \%)$ patient, uveitis in $7(15.55 \%)$, Hepatitis in $4(9.3 \%)$, convulsion in $2(4.6 \%)$ and $1(2.3 \%)$ patient each experienced aseptic meningitis and hearing loss. Regarding outcome of the patient 39 patient $(86 \%)$ were improved. Two patient $(4.44 \%)$ left against medical advice and one patient died (Table-8).

\section{DISCUSSION}

Kawasaki disease (KD) is an acute vasculitis of childhood with a special predilection to involve coronary arteries. If untreated, it can lead to coronary artery aneurysms in $25 \%$ of cases [10]. It was previously called mucocutaneous lymph node syndrome and infantile periarteritisnodosa [11]. In countries like Japan, the United States, and certain European countries, $\mathrm{KD}$ is now considered as the most common acquired heart disease in children [11]. Kawasaki disease is an acute multisystem vasculitis of unknown etiology that occurs in children of all races, but is more common in Japan (up to 175 per 100,000) [10]. The incidence of Kawasaki disease is increasing worldwide [12]. It has surpassed rheumatic fever as the leading cause of acquired heart disease in children according to some studies [13]. This may be males are more prone to develop Kawasaki disease or may be because socially females are still neglected while seeking healthcare even in serious health issues in perspective of Bangladesh. In Japanese children, the incidence of KD is highest between 6 and 12 months of age but in our 
study highest incidence of Kawasaki disease was observed among children of 1 to 2years of age $(20.68 \%)$. In this study all of the study patients $(n=45$ or $100 \%$ ) presented with fever with mean duration was $12 \pm 3$ days. In studies by Akhtar et al and Singh et al fever was present in $100 \%$ of the patients. Non purulent conjunctivitis (97\%) was the second most commonly observed symptoms in this study. In a study by sayeed et al., $100 \%$ of patient had bilateral conjunctivitis and Burns et al shows $70 \%$ patient had anterior uveitis. In this study unilateral cervical lymphadenopathy (51\%) was the least observed feature which was set as one of the cardinal feature for diagnosing Kawasaki disease and it is similar to the study by Sayeed et al where lymphadenopathy was found only in $68 \%$. The causative agent still remains unclear. $^{14}$ No study addressing cardiovascular involvement in Kawasaki disease among pediatric age group has been published from Bangladesh. According to some international studies, males are generally affected more from KD than the females $[15,16]$. Another study from India showed that $\mathrm{KD}$ is definitely being increasingly recognized and reported in India [18]. This could be either due to an actual rise in number of cases or due to increased awareness amongst pediatricians. Many physicians and pediatricians are of the view that rise in KD coincided with the fall of incidence of diarrhea and better vaccination coveragerates [19]. A study from Hong Kong also showed an increase in incidence from 26 per 100,000 children <5 years in 1994 to 39 per 100,000 in 2000 and to 74 per 100,000 in 2011 [17]. In this study it is also observed that male child are more affected $(72 \%)$ by Kawasaki disease. However, in the USA and Europe, the peak age group for KD is 18-24 months [19]. In Kawasaki disease, fever occurs because of the elevated levels of different proinflammatory cytokines, which are also thought to mediate the underlying vascular inflammation affecting the cardiovascular system. Cardiac involvement is the most important feature of Kawasaki disease. Cardiac involvement has been variably reported in up to $25 \%$ of the cases and coronary artery involvement was seen in $41 \%$ of our cases [20]. In this study $42.22 \%$ of the patients $(n=19)$ showed features of coronary involvement. Involvement of the left main coronary artery (LMCA) was most observed coronary change found in $68 \%$ with mean size $2.9 \pm 0.47 \mathrm{~mm}$ and least was left circumflex artery (LCX) change (10.5\%) of patients having coronary change. A study by Saleem et al., also showed left main coronary artery, left anterior descending coronary artery and right coronary artery are more likely to be involved and circumflex branch is least commonly involved which is more or like consistent with our present study. Furusho et al., described the role of IVIG treatment which became standard of care. The benefits of higher doses of IVIG with aspirin was demonstrated in a review of 1629 patients with KD from six randomized controlled studies [21]. In this study also 93\% of the patient received intravenous immunoglobulin and $100 \%$ patient high dose of aspirin in acute phase. The commonly observed complication was uveitis observed in $17.77 \%$ $(n=45)$ and $6.66 \%$ patient had IVIG resistant Kawasaki disease. A study conducted by Choi et al in Korea also revealed uveitis as common complication and most important criteria for diagnosing Kawasaki disease. As for many physicians in developing countries like Bangladesh, where the burden of infectious disease is high, $\mathrm{KD}$ is still not commonly included in the differential diagnosis of children presenting with fever. Some of the cardinal manifestations of KD (e.g., fever, rash and lymphadenitis) are also seen in many pediatric infectious diseases and it is not surprising that KD gets overlooked in such a milieu. Therefore, pediatricians in developing countries need to be sensitized about KD.

\section{CONCLUSION AND RECOMMENDATIONS}

Kawasaki disease is now fast emerging as one of the important cause of acquired heart disease and significant contributory factor to the long-term cardiac morbidity and mortality in these patients. Therefore, clinicians should have a high index of suspicion of Kawasaki disease in persistently febrile patients and once clinically diagnosed, echocardiography should be done and IVIG therapy along with aspirin should be started, especially in infants ( $<6$ months) as they have the higher tendency to develop coronary aneurysm. Authors are recommending multi-centre study with large sample size.

\section{REFERENCES}

1. Patel, A., Holman, R. C., Callinan, L. S., Sreenivasan, N., Schonberger, L. B., Fischer, T. K., $\&$ Belay, E. D. (2013). Evaluation of clinical characteristics of Kawasaki syndrome and risk factors for coronary artery abnormalities among children in Denmark. Acta Paediatrica, 102(4), 385-390.

2. Callinan, L. S., Tabnak, F., Holman, R. C., Maddox, R. A., Kim, J. J., Schonberger, L. B., ... \& Belay, E. D. (2012). Kawasaki syndrome and factors associated with coronary artery abnormalities in California. The Pediatric infectious disease journal, 31(9), 894-898.

3. Taubert, K. A., Rowley, A. H., \& Shulman, S. T. (1994). Seven-year national survey of Kawasaki disease and acute rheumatic fever. The Pediatric infectious disease journal, 13(8), 704-708.

4. Falcini, F., Capannini, S., \& Rigante, D. (2011). Kawasaki syndrome: an intriguing disease with numerous unsolved dilemmas. Pediatric rheumatology, 9(1), 1-8.

5. Newburger, J. W., Takahashi, M., Gerber, M. A., Gewitz, M. H., Tani, L. Y., Burns, J. C., ... \& Taubert, K. A. (2004). Diagnosis, treatment, and long-term management of Kawasaki disease: a statement for health professionals from the Committee on Rheumatic Fever, Endocarditis and Kawasaki Disease, Council on Cardiovascular Disease in the Young, American Heart 
Association. Circulation, 110(17), 2747-2771.

6. Eleftheriou, D., Levin, M., Shingadia, D., Tulloh, R., Klein, N. J., \& Brogan, P. A. (2014). Management of Kawasaki disease. Archives of disease in childhood, 99(1), 74-83.

7. Merchechi, A., Tarissi, I., Marucci, G., \& Villani, A. (2015). Typical Kawasaki disease. Italian Journal of Pediatr, 41(suppl 2), A47.

8. Singh, S., Agarwal, S., Bhattad, S., Gupta, A., Suri, D., Rawat, A., ... \& Rohit, M. (2016). Kawasaki disease in infants below 6 months: a clinical conundrum?. International journal of rheumatic diseases, 19(9), 924-928.

9. Newburger, J. W., Takahashi, M., Gerber, M. A., Gewitz, M. H., Tani, L. Y., Burns, J. C., ... \& Taubert, K. A. (2004). Diagnosis, Treatment, and Long-term Management of Kawasaki Disease: A Statement forHealth Professionals From the Committee on RheumaticFever, Endocarditis, and Kawasaki Disease, Council onCardiovascular Disease in the Young, American Heart Association. Pediatrics, 114, 1708-1733.

10. Jiao, F., Jindal, A. K., Pandiarajan, V., Khubchandani, R., Kamath, N., Sabui, T., ... \& Singh, S. (2017). The emergence of Kawasaki disease in India and China. Global cardiology science \& practice, 2017(3), e201721.

11. Holman, R. C., Belay, E. D., Christensen, K. Y., Folkema, A. M., Steiner, C. A., \& Schonberger, L. B. (2010). Hospitalizations for Kawasaki syndrome among children in the United States, 19972007. The Pediatric infectious disease journal, 29(6), 483-488.

12. Zhu, H., Yu, S. F., Bai, Y. X., Liang, Y. Y., Su, X. W., \& Pan, J. Y. (2015). Kawasaki disease in children: Epidemiology, clinical symptoms and diagnostics of 231 cases in 10 years. Experimental and therapeutic medicine, 10(1), 357-361.

13. Kato, H., Sugimura, T., Akagi, T., Sato, N., Hashino, K., Maeno, Y., ... \& Yamakawa, R. (1996). Long-term consequences of Kawasaki disease: a 10-to 21-year follow-up study of 594 patients. Circulation, 94(6), 1379-1385.

14. Taubert, K. A., Rowley, A. H., \& Shulman, S. T. (1991). Nationwide survey of Kawasaki disease and acute rheumatic fever. The Journal of pediatrics, 119(2), 279-282.

15. Harada, K., Yamaguchi, H., Kato, H., Nishibiyashi, Y., Ischiro, S., Okazaki, T., Sato, Y., Furusho, K., Okawa, S., \& Kawasaki, T. (1993). Indication for intravenous gamma globulin treatment for Kawasaki disease: In Proceedings of the Fourth International Symposium on Kawasaki Disease. Edited by Takahashi M, Taubert K Dallas, Tex: American Heart Association; 459-462.

16. Beiser, A. S., Takahasha, M., Baker, A. L., Sundel, R. P., \& Newburger, J. W. (1998). United States Multicenter Kawasaki Disease Study Group: A predictive instrument for coronary artery aneurysms in Kawasaki disease. Am J Cardiol, 81, 1116-1120.

17. Forsey, J., \& Mertens, L. (2012). Atypical Kawasaki disease - a clinical challenge. European journal of pediatrics, 171(4), 609-611.

18. Marsechi, A., Pongiglioni, G., Rimini, A., Longhi, R., \& Villani A. (2008). Malattia di Kawasaki: Linee Guidaitaliani. Prospettive in Pediatria, 38, 266-283.

19. Rowley, A. H., \& Shulman, S. T. (1998). Kawasaki syndrome. Clinical microbiology reviews, 11(3), 405-414.

20. Cabral, M., Correia, P., Brito, M. J., Conde, M., \& Carreiro, H. (2011). Kawasaki disease in a young infant: diagnostic challenges. Acta reumatologica portuguesa, 304-308.

21. Tsuchida, S., Yamanaka, T., Tsuchida, R., Nakamura, Y., Yashiro, M., \& Yanagawa, H. (1996). Epidemiology of infant Kawasaki disease with a report of the youngest neonatal case ever reported in Japan. Acta Paediatrica, 85(8), 995 997. 